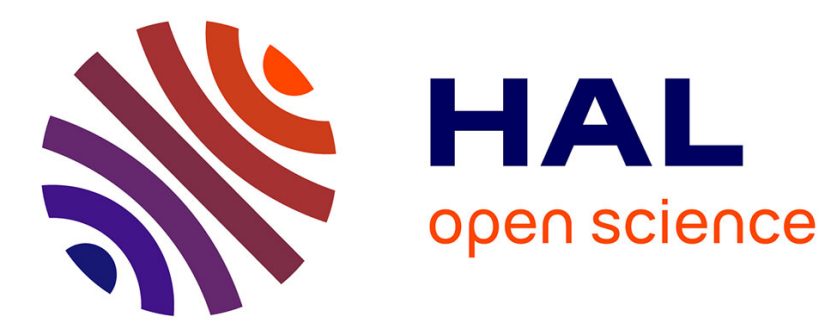

\title{
Multidimensional asymptotic spectral analysis and applications
}

\author{
Victor Devoue, Maximilian F. Hasler, Jean-André Marti
}

\section{To cite this version:}

Victor Devoue, Maximilian F. Hasler, Jean-André Marti. Multidimensional asymptotic spectral analysis and applications. 2010. hal-00761112

\section{HAL Id: hal-00761112 https://hal.univ-antilles.fr/hal-00761112}

Preprint submitted on 4 Dec 2012

HAL is a multi-disciplinary open access archive for the deposit and dissemination of scientific research documents, whether they are published or not. The documents may come from teaching and research institutions in France or abroad, or from public or private research centers.
L'archive ouverte pluridisciplinaire HAL, est destinée au dépôt et à la diffusion de documents scientifiques de niveau recherche, publiés ou non, émanant des établissements d'enseignement et de recherche français ou étrangers, des laboratoires publics ou privés. 


\section{Document de travail 2010-03}

Mai 2010

\section{"Multidimensional asymptotic spectral analysis and applications"}

Victor DEVOUE, Maximilian HASLER \& Jean-André MARTI 


\title{
Multidimensional asymptotic spectral analysis and applications
}

\author{
V. Dévoué, M. F. Hasler \& J.-A. Marti* \\ Laboratoire CEREGMIA, Université des Antilles et de la Guyane \\ Campus de Schoelcher, Martinique, France \\ * E-mail: jamarti@univ-ag.fr
}

\begin{abstract}
This article adresses questions on localization and microlocalization in the framework of the so called $(\mathcal{C}, \mathcal{E}, \mathcal{P})$-algebras of generalized functions. We give new results on nonlinear properties of the asymptotic singular spectrum in the multi-parameter case, a tool to describe the singularities of generalized solutions associated to problems which are classically ill-posed. Wa apply the theory to a transport equation with strongly irregular coefficients and initial data.

Keywords: Algebras of generalized functions, local and microlocal asymptotic analysis, transport equation with irregular coefficients and irregular data.

MSC-class: 46F30, 46T30, 35D99.
\end{abstract}

\section{Introduction}

This article adresses questions on localization and microlocalization in the framework of the presheaf $\mathcal{A}$ over a topological space $X$ of so called $(\mathcal{C}, \mathcal{E}, \mathcal{P})$-algebras $[7,8]$. These algebras extend the structure of some various types of generalized functions developped over the past twenty years by many authors. A review of the ideas, technics and results on microlocalization is given in [9]. It is dissociated into a (classical) frequential microlocal analysis based on the Fourier transform and into a (more recent) microlocal asymptotic analysis described and studied in [4], which has new properties with respect to nonlinear operations. In [4], the spectral analysis is linked to the complement of a "regular fiber" which is a subset of $\mathbb{R}_{+}$.

However, some differential problems with several independant singularities in coefficient or data need a multidimentional analysis of the solutions. Consequently in Section 2, the "regular fiber" will be a subset of $\mathbb{R}_{+}^{n}$ and the definitions and results of [4] are extended to the case of multidimensional asymptotic analysis. If $\mathcal{F}$ is given sheaf of topological vector space over $X, \Omega$ an open set in $X$, we begin to define the $\mathcal{F}$-singular support of some generalized function $u \in \mathcal{A}(\Omega)$. Moreover when $a$ is a special "analyzing" function, the $(a, \mathcal{F})$-singular spectrum of $u$ gives a spectral analysis of the singularities meaning where and why $u$ is not locally (associated to a section of) $\mathcal{F}$. The desciption of such a spectrum and its behavior under linear or nonlinear 
operations are also given in this section.

In section 3, we give an example of asymptotic analysis. Various cases of transport equation with irregular coefficient (lying generally in Sobolev spaces) are studied in the literature (see $[1,6]$ ). We study here the Cauchy problem for transport equation with stronger singularites in the case where the following coefficients $\alpha$ and $\beta$ are distributions. For the data $u_{0}$, we suppose it is a distribution and even a more singular object like $\delta_{x}^{p} \otimes \delta_{y}^{q}$ we will give later a generalized meaning. Then the problem is formally written as

$$
\left(\mathcal{P}_{\text {form }}\right)\left\{\begin{array}{r}
\frac{\partial}{\partial t} u+\alpha \otimes 1_{x y} \frac{\partial}{\partial x} u+\beta \otimes 1_{x y} \frac{\partial}{\partial y} u=0, \\
\left.u\right|_{\{t=0\}}=u_{0}\left(=\delta_{x}^{p} \otimes \delta_{y}^{q}\right) .
\end{array}\right.
$$

The product and the restriction written above are generally not defined in a distributional sense. Consequently we begin in associating to $\left(\mathcal{P}_{\text {form }}\right)$ a generalized one $\left(\mathcal{P}_{\text {gen }}\right)$ well formulated in a convenient $(\mathcal{C}, \mathcal{E}, \mathcal{P})$ algebra $\mathcal{A}\left(\mathbb{R}^{3}\right)$ constructed by means of independant regularizations involving three independent parameters $\varepsilon, \eta, \rho$ and obtain

$$
\left(\mathcal{P}_{\text {gen }}\right)\left\{\begin{array}{r}
\frac{\partial}{\partial t} u+F \frac{\partial}{\partial x} u+G \frac{\partial}{\partial y} u=0 \\
\left.u\right|_{\{t=0\}}=H .
\end{array}\right.
$$

where $F$ and $G$ (resp. $H$ ) are the classes in $\mathcal{A}\left(\mathbb{R}^{3}\right)$ (resp. $\mathcal{A}\left(\mathbb{R}^{2}\right)$ ) of the families regularizing the coefficients (resp. the data).

First we solve $\left(\mathcal{P}_{\text {gen }}\right)$ and examine the existence of a solution. The asymptotic singular spectrum defined in Section 2 is adapted here to the three-parametric case by choosing $\mathcal{F}=\mathcal{D}^{\prime}$ and $a\left(r_{1}, r_{2}, r_{3}\right)=\varepsilon^{r_{1}} \eta^{r_{2}} \rho^{r_{3}}$. Then the " $\left(a, \mathcal{D}^{\prime}\right)$-singular spectrum" of $u \in \mathcal{A}\left(\mathbb{R}^{3}\right)$ gives a spectral analysis of the singularities. The localization of such singularities of $u$ is always the " $\mathcal{D}^{\prime}$-singular support" of $u$, and the asymptotic causis is described by a fiber $\Sigma_{X}(u)$ (above each $X=(t, x, y) \in \mathbb{R}^{3}$ ) which is the complement in $\mathbb{R}_{+}^{3}$ of a conic subset of $\mathbb{R}_{+}^{3}$.

In our case, the $\mathcal{D}^{\prime}$-singularities of the data propagate along the "regularized characteristic $\Gamma$ of the problem $\left(\mathcal{P}_{g e n}\right)$ " on which the fiber $\Sigma_{X}(u)$ remains constant.

\section{The $(\mathcal{C}, \mathcal{E}, \mathcal{P})$-structure, localization and microlo- calization processes}

\subsection{The algebraic structure}

We suppose that

- $\mathbb{K}$ is the real or complex field, and $\Lambda$ a set of indices, left-filtering for a partial order $\prec$.

- $\mathcal{C}$ is a factor ring $A / I$ where $I$ is an ideal of $A$, a given subring of $\mathbb{K}^{\Lambda}$. 
- $A$ and $I$ are both solid, i.e., equal to their solid hull,

$$
\operatorname{sh}(A)=\left\{x \in \mathbb{K}^{\Lambda}|\exists a \in A, \forall \lambda \in \Lambda:| x_{\lambda}|\leq| a_{\lambda} \mid\right\} .
$$

- $(\mathcal{E}, \mathcal{P})$ is a sheaf of topological $\mathbb{K}$-algebras on a topological space $X$, the topology on $\mathcal{E}(\Omega)$ being given, for any open set $\Omega$ in $X$, by a family $\mathcal{P}(\Omega)$ of semi-norms.

Then we set

$$
\begin{aligned}
\mathcal{H}_{(A, \mathcal{E}, \mathcal{P})}(\Omega) & =\left\{\left(u_{\lambda}\right)_{\lambda} \in[\mathcal{E}(\Omega)]^{\Lambda} \mid \forall p \in \mathcal{P}(\Omega),\left(p\left(u_{\lambda}\right)\right)_{\lambda} \in A\right\}, \\
\mathcal{J}_{(I, \mathcal{E}, \mathcal{P})}(\Omega) & =\left\{\left(u_{\lambda}\right)_{\lambda} \in[\mathcal{E}(\Omega)]^{\Lambda} \mid \forall p \in \mathcal{P}(\Omega),\left(p\left(u_{\lambda}\right)\right)_{\lambda} \in I\right\} .
\end{aligned}
$$

Under some more technical conditions detailed in [4], we have

Theorem 1 The factor space $\mathcal{A}=\mathcal{H}_{(A, \mathcal{E}, \mathcal{P})} / \mathcal{J}_{(I, \mathcal{E}, \mathcal{P})}$ is a presheaf with localization principle.

The proof is given in [4]

Definition $1 \mathcal{A}$ is said a presheaf of $(\mathcal{C}, \mathcal{E}, \mathcal{P})$-algebras. The equivalence class in $\mathcal{A}(\Omega)$ of $\left(u_{\lambda}\right)_{\lambda \in \Lambda} \in \mathcal{H}_{(A, \mathcal{E}, \mathcal{P})}(\Omega)$ is denoted by $\left[u_{\lambda}\right]$. For $u \in \mathcal{A}(\Omega)$, the notation $\left(u_{\lambda}\right)_{\lambda \in \Lambda} \in u$ means that $\left(u_{\lambda}\right)_{\lambda \in \Lambda}$ is a representative of $u$.

\subsection{Overgenerated algebras}

The following construction provides a systematic method of constructing a subring $A$ and its ideal $I$, suitable for dealing with a given differential problem.

Definition 2 Consider a family $B$ of nets in $\left(\mathbb{R}_{+}^{*}\right)^{\Lambda}$. Let $\langle B\rangle$ be the closure of $B$ in $\left(\mathbb{R}_{+}^{*}\right)^{\Lambda}$ with respect to addition and division; $\langle B\rangle$ is given as the subset of elements in $\left(\mathbb{R}_{+}^{*}\right)^{\Lambda}$ obtained as rational fractions of elements in $B$ with coefficients in $\mathbb{N}^{*}$, Now let

$$
A=\left\{\left(a_{\lambda}\right)_{\lambda} \in \mathbb{K}^{\Lambda}\left|\exists\left(b_{\lambda}\right)_{\lambda} \in\langle B\rangle, \exists \lambda_{0} \in \Lambda, \forall \lambda \prec \lambda_{0}:\right| a_{\lambda} \mid \leq b_{\lambda}\right\} .
$$

Then we say that $A$ is overgenerated by $B$. An ideal of $A$ is given by

$$
I_{A}=\left\{\left(a_{\lambda}\right)_{\lambda} \in \mathbb{K}^{\Lambda}\left|\forall\left(b_{\lambda}\right)_{\lambda} \in\langle B\rangle, \exists \lambda_{0} \in \Lambda, \forall \lambda \prec \lambda_{0}:\right| a_{\lambda} \mid \leq b_{\lambda}\right\} .
$$

Remark 1 It is easy to see that $A$ and $I_{A}$ are solid subrings of $\mathbb{K}^{\Lambda}$. By definition, $\langle B\rangle$ is stable by inverse, This also implies that $I_{A}$ is the set of all nets dominated by all elements of $A^{*}$, the elements of $A$ having an inverse in $A$. This is a general way of associating a "canonical" ideal $I_{A}$ to a given subring $A$. (The requirement of having an inverse in $A$ ensures that the elements are not arbitrarily small.) 


\subsection{Association processes}

In all what follows, we will assume that all nets $\left(j_{\lambda}\right) \in I$ have zero limit. Then also any representative $\left(v_{\lambda}\right) \in o=\mathcal{J}_{I, \mathcal{E}, \mathcal{P}}$ will have zero limit in $\mathcal{E}$.

Definition 3 If $\mathcal{F}$ is a presheaf of topological $\mathbb{K}$-vector spaces which contains $\mathcal{E}$ as a subsheaf, and $a \in \mathcal{C}$ is a generalized number, then we say that $u \in \mathcal{A}(\Omega)$ is aassociated to $f \in \mathcal{F}(\Omega)$ iff some (and thus all) representatives of the generalized function a $u \in \mathcal{A}_{(\mathcal{C}, \mathcal{E}, \mathcal{P})}$ have $f$ as a limit in $\mathcal{F}(\Omega)$ :

$$
u \underset{\mathcal{F}(\Omega)}{a} f \Longleftrightarrow \lim _{\Lambda}\left(\left.a_{\lambda} u_{\lambda}\right|_{\Omega}\right)=f \in \mathcal{F}(\Omega)
$$

In particular, if this holds with $a=1$, then $u$ and $f$ are said associated in $\Omega$.

Example 1 Taking $X=\mathbb{R}^{d}, \mathcal{F}=\mathcal{D}^{\prime}, \Lambda=(0,1], \mathcal{A}=\mathcal{G}$, the usual association between $u=\left[u_{\varepsilon}\right] \in \mathcal{G}(\Omega)$ and $T \in \mathcal{D}^{\prime}(\Omega)$ is obtained for $a=1$,

$$
u \sim T \Longleftrightarrow u \underset{\mathcal{D}^{\prime}(\Omega)}{\stackrel{1}{\sim}} T \Longleftrightarrow \lim _{\varepsilon \rightarrow 0} \mathcal{D}^{\prime}(\Omega) u_{\varepsilon}=T
$$

The definition also gives rise to the subspace $\mathcal{J}_{(a, \mathcal{F})}$ of elements a-associated to o $\in$ $\mathcal{F}(\Omega)$, and to the related association among two elements $u, v$ of $\mathcal{A}(\Omega)$,

$$
u \underset{\mathcal{F}}{\stackrel{a}{\widetilde{F}}} v \Longleftrightarrow u-v \underset{\mathcal{F}}{\stackrel{a}{\widetilde{F}}} o_{\mathcal{F}}
$$

If we take $a=\left[\left(\varepsilon^{-p}\right)_{\varepsilon}\right]$, we get the p-association introduced in [8].

Proposition 2 If $u \in \mathcal{A}(\Omega)$ is a-associated to $f \in \mathcal{F}(\Omega)$, then $u$ is $a^{\prime}$-associated to $o \in \mathcal{F}(\Omega)$ for all $a^{\prime}=o(a)$, i.e., having a representative $\left(a_{\lambda}^{\prime}\right)=o\left(a_{\lambda}\right)$.

Proof. If $u \underset{\mathcal{F}(\Omega)}{\stackrel{a}{f}} f$ and $\left(a_{\lambda}^{\prime}\right)=o\left(a_{\lambda}\right)$, then $\lim a_{\lambda} u_{\lambda}=f \in \mathcal{F}(\Omega)$ and $a_{\lambda}^{\prime}=\eta_{\lambda} a_{\lambda}$ with $\lim \eta_{\lambda}=0$; thus $\lim a_{\lambda}^{\prime} u_{\lambda}=\lim \eta_{\lambda} a_{\lambda} u_{\lambda}=\lim \eta_{\lambda} f=o \in \mathcal{F}(\Omega)$, whence $u \underset{\mathcal{F}(\Omega)}{\stackrel{a^{\prime}}{\sigma}} o$.

\subsection{Localization of $\mathcal{F}$-singularities: asymptotic singular sup- port}

The previous considerations lead us in a natural way to

Definition 4 - To any presheaf $\mathcal{F}$ of topological $\mathbb{K}$-vector spaces which contain the subsheaf $\mathcal{E}$, we associate the following subsheaf of $\mathcal{A}$,

$$
\mathcal{F}_{\mathcal{A}}(\Omega)=\left\{u \in \mathcal{A}(\Omega) \mid \exists\left(u_{\lambda}\right)_{\lambda} \in u, \exists f \in \mathcal{F}(\Omega): \lim _{\Lambda} \mathcal{F}(\Omega) u_{\lambda}=f\right\} .
$$


- This leads to the open set (of points) of $\mathcal{F}$-regularity of a given section $u \in \mathcal{A}$,

$$
\mathcal{O}_{\mathcal{A}}^{\mathcal{F}}(u)=\left\{x \in \Omega\left|\exists V \in \mathcal{V}_{x}: u\right|_{V} \in \mathcal{F}_{\mathcal{A}}(V)\right\},
$$

where $\mathcal{V}_{x}$ denotes the set of all the neighborhoods of $x$

The complement of $\mathcal{O}_{\mathcal{A}}^{\mathcal{F}}(u)$ on $\Omega$, is called the $\mathcal{F}$-singular support of $u \in \mathcal{A}(\Omega)$ :

$$
\mathcal{S}_{\mathcal{A}}^{\mathcal{F}}(u)=\Omega \backslash \mathcal{O}_{\mathcal{A}}^{\mathcal{F}}(u) .
$$

It is clear that $\mathcal{S}_{\mathcal{A}}^{\mathcal{F}}(u)$ is a closed subset contained in $\operatorname{supp}(u)$.

\subsection{Microlocalization of $\mathcal{F}$-singularities: asymptotic singular spectrum}

The idea of the $(\mathbf{a}, \mathcal{F})$-microlocal analysis is the following.

If $u=\left[u_{\lambda}\right] \in \mathcal{A}(\Omega)$ is not in $\mathcal{F}_{\mathcal{A}}$ in a given point $x \in \Omega$, i.e., there is no $V \in \mathcal{V}_{x}$ and $f \in \mathcal{F}(V)$ such that $\lim _{\Lambda} u_{\lambda}=f$ in $\mathcal{F}(V)$, there are nevertheless generalized numbers $a \in \mathcal{C}=A / I$ "small enough" such that $a u \in \mathcal{F}_{\mathcal{A}}(V)$ in some neighborhood $V$ of $x$. (In particular, this is true for $a=0$.)

More precisely, we can consider a map a : $\mathbb{R}_{+}^{n} \rightarrow \mathcal{C}=A / I$ with some convenient properties, such that the set of $r \in \mathbb{R}_{+}^{n}$ for which $\mathbf{a}(r) u \in \mathcal{F}_{\mathcal{A}}$, will give more detailed information of the "roughness" of $u$ at a given point.

These considerations lead to the following definition which generalizes the corresponding one in [4], where $\mathbf{a}$ is a map from $\mathbb{R}_{+}$to $A_{+}$, instead of $\mathbb{R}_{+}^{n}$ to $\mathcal{C}$ as here:

Definition 5 Assume that

- $\mathcal{F}$ is a given presheaf of topological $\mathbb{K}$-vector spaces over $X$ containing $\mathcal{E}$ as a subsheaf,

- a is a map from $\mathbb{R}_{+}^{n}$ to $\mathcal{C}$ such that $\mathbf{a}(o)=1_{\mathcal{C}}$, and

$$
\forall r, s \in \mathbb{R}_{+}^{n} \backslash\{o\}: \mathbf{a}(r)=o(1) \text { and } \mathbf{a}(r+s)=O(\mathbf{a}(r) \mathbf{a}(s))
$$

where $\mathbf{a}(r)=\left[\mathbf{a}_{\lambda}(r)\right]$, with $r=\left(r_{1}, r_{2}, \ldots r_{n}\right) \in \mathbb{R}_{+}^{n}$, and

$$
\mathbf{a}^{\prime}=O(\mathbf{a}) \Longleftrightarrow \exists C>0,\left(\mathbf{a}_{\lambda}^{\prime}\right) \in \mathbf{a}^{\prime},\left(\mathbf{a}_{\lambda}\right) \in \mathbf{a}, \forall \lambda \in \Lambda:\left|\mathbf{a}_{\lambda}^{\prime}(r)\right| \leq C\left|\mathbf{a}_{\lambda}(r)\right| .
$$

Then we define, for any open subset $\Omega$ of $X, u=\left[u_{\lambda}\right] \in \mathcal{A}(\Omega)$ and $x \in \Omega$, the $(a, \mathcal{F})$-regular fiber of $u$ over $x$ as the set

$$
\begin{aligned}
& N_{(\mathbf{a}, \mathcal{F}), x}(u)=\left\{r \in \mathbb{R}_{+}^{n} \mid \exists V \in \mathcal{V}_{x}, \exists f \in \mathcal{F}(V): \lim _{\Lambda} \mathcal{F}(V)\right. \\
&\left.=\left\{r \in \mathbb{R}_{+}^{n}\left|\exists V \in \mathcal{a}_{\lambda}(r) u_{\lambda}\right|_{V}\right)=f\right\} \\
&\left.\left.\mathbf{a}(r) u\right|_{V} \in \mathcal{F}_{\mathcal{A}}(V)\right\}
\end{aligned}
$$

and the $(a, \mathcal{F})$-singular fiber of $u$ over $x$ as its complement in $R_{+}^{n}$,

$$
\Sigma_{(\mathbf{a}, \mathcal{F}), x}(u)=\mathbb{R}_{+}^{n} \backslash N_{(\mathbf{a}, \mathcal{F}), x}(u) .
$$


From the requirement that $x \in I \Rightarrow \lim \left(x_{\lambda}\right)=0$, the definitions of $N_{(\mathbf{a}, \mathcal{F}), x}(u)$ and $\Sigma_{(\mathbf{a}, \mathcal{F}), x}(u)$ don't depend on the representative of $u$.

When $(\mathbf{a}, \mathcal{F})$ can be considered to be given, we shall write for short,

$$
N_{(\mathbf{a}, \mathcal{F}), x}(u)=N_{x}(u), \quad \Sigma_{(\mathbf{a}, \mathcal{F}), x}(u)=\Sigma_{x}(u) .
$$

In other terms, for each $r \in N_{x}(u)$ (resp. $r \in \Sigma_{x}(u)$ ), the generalized function $u$ is (resp. cannot be) locally a $(r)$-associated to a section of $\mathcal{F}$ above $x$.

Remark 2 Obviously, $\Sigma_{x}(u)=\emptyset \Longleftrightarrow o \in N_{x}(u) \Longleftrightarrow x \notin \operatorname{sing} \operatorname{supp}_{\mathcal{F}}(u)$ since this means that there is a neighborhood $V$ of $x$ such that $\left.u\right|_{V} \in \mathcal{F}_{\mathcal{A}}(V)$.

Theorem 3 - The $(a, \mathcal{F})$-regular fiber $N_{x}(u)$ is the (possibly empty) union $\bigcup_{r \in N_{x}(u)} \Gamma_{r}$ of closed cones

$$
\Gamma_{r}=\prod_{i=1}^{n}\left[r_{i},+\infty\right)=r+\mathbb{R}_{+}^{n}
$$

- The $(a, \mathcal{F})$-singular fiber $\Sigma_{x}(u)$ is either $\mathbb{R}_{+}^{n}$, or $\bigcap_{r \in N_{x}(u)}\left(\mathbb{R}_{+}^{n} \backslash \Gamma_{r}\right)$.

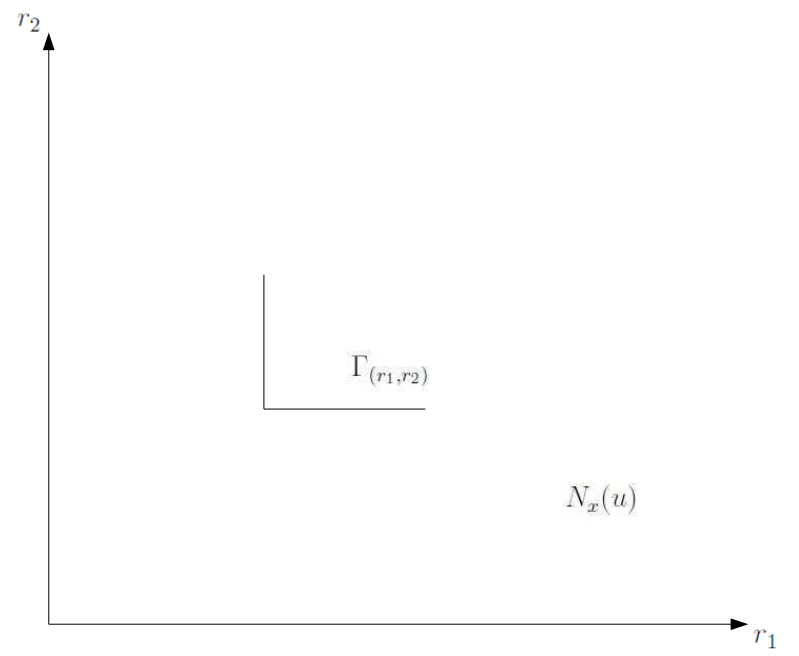

Proof. We have to show that $r \in N_{x}(u) \Rightarrow r+s \in N_{x}(u)$ for all $s \in \mathbb{R}_{+}^{n}$. Then, with any point $r, N_{x}(u)$ also contains the cone $r+\Gamma_{r}$, and $N_{x}(u)$ equals the union of these cones as claimed.

Assume that $r \in N_{x}(u)$, i.e., $\left.\mathbf{a}(r) u\right|_{V} \in \mathcal{F}_{\mathcal{A}}(V)$ for some neighborhood $V$ of $x$. For $s=o$, there is nothing to show, and for $s \neq o$, we have $\mathbf{a}(s)=o(1)$ and thus $\mathbf{a}(r+$ $s)=O(\mathbf{a}(r) \mathbf{a}(s))=o(1 \mathbf{a}) a(r)$, whence $\left.\mathbf{a}(r+s) u\right|_{V}=\left.o(1) \mathbf{a}(r) u\right|_{V} \in \mathcal{F}_{\mathcal{A}}(V)$ as in Proposition 2. 
Definition 6 The $(\mathbf{a}, \mathcal{F})$-singular spectrum of $u \in A(\Omega)$ is the set

$$
\mathcal{S}_{\mathcal{A}}^{(\mathbf{a}, \mathcal{F})}(u)=\left\{(x, r) \in \Omega \times \mathbb{R}_{+}^{n} \mid r \in \Sigma_{x}(u)\right\} .
$$

Theorem 4 The projection of the $(a, \mathcal{F})$-singular spectrum of $u$ on $\Omega$ is the $\mathcal{F}$ singular support of $u$,

$$
\left\{x \in \Omega \mid \exists r \in \mathbb{R}_{+}^{n}:(x, r) \in \mathcal{S}_{\mathcal{A}}^{(\mathbf{a}, \mathcal{F})}(u)\right\}=\mathcal{S}_{\mathcal{A}}^{\mathcal{F}}(u) .
$$

Proof. This is an immediate consequence of Remark 2. Indeed, $\exists r:(x, r) \in \mathcal{S}_{\mathcal{A}}^{(\mathbf{a}, \mathcal{F})}(u) \Longleftrightarrow \Sigma_{x}(u) \neq\left.\emptyset \Longleftrightarrow o \notin N_{x}(u) \Longleftrightarrow u\right|_{V} \notin \mathcal{F}_{\mathcal{A}}(V)$.

\subsection{Linear and differential properties of the $(a, \mathcal{F})$-singular asymp- totic spectrum}

It is easy to prove that for any $u, v \in \mathcal{A}(\Omega)$, we have

$$
\mathcal{S}_{\mathcal{A}}^{(\mathbf{a}, \mathcal{F})}(u+v) \subset \mathcal{S}_{\mathcal{A}}^{(\mathbf{a}, \mathcal{F})}(u) \cup \mathcal{S}_{\mathcal{A}}^{(\mathbf{a}, \mathcal{F})}(v) .
$$

Assume that $\mathcal{F}$ is a presheaf of topological differential vector spaces, with continuous differentiation, admitting $\mathcal{E}$ as a subsheaf of topological differential algebras. Then the presheaf $\mathcal{A}$ is also a presheaf of differential algebras with, for any $\alpha \in \mathbb{N}^{d}$ and $u \in \mathcal{A}(\Omega)$,

$$
\partial^{\alpha} u=\left[\partial^{\alpha} u_{\lambda}\right], \text { where }\left(u_{\lambda}\right)_{\lambda} \text { is any representative of } u .
$$

Assume in addition that $\mathcal{F}$ is a presheaf of topological modules over $\mathcal{E}$. This leads to following statement:

\section{Theorem 5 Let}

$$
P(\partial)=\sum_{|\alpha| \leq m} C_{\alpha} \partial^{\alpha}
$$

be a differential polynomial with coefficients in $\mathcal{E}(\Omega)$. For any $u \in A(\Omega)$, we have

$$
\mathcal{S}_{\mathcal{A}}^{(\mathbf{a}, \mathcal{F})}(P(\partial) u) \subset \mathcal{S}_{\mathcal{A}}^{(\mathbf{a}, \mathcal{F})}(u) .
$$

The proof is given in [4]

\subsection{Nonlinear properties}

When $\mathcal{F}$ is a presheaf of algebras, the $(\mathbf{a}, \mathcal{F})$-singular spectrum inherits new properties with respect to nonlinear operations. It is the subject of the following results.

Theorem 6 For given $u$ and $v \in \mathcal{A}(\Omega)$, let $D_{i}(i=1,2,3)$ be the following disjoint sets:

$$
D_{1}=\mathcal{S}_{\mathcal{A}}^{\mathcal{F}}(u) \backslash D_{3} ; \quad D_{2}=\mathcal{S}_{\mathcal{A}}^{\mathcal{F}}(v) \backslash D_{3} ; \quad D_{3}=\mathcal{S}_{\mathcal{A}}^{\mathcal{F}}(u) \cap \mathcal{S}_{\mathcal{A}}^{\mathcal{F}}(v) .
$$


Then, the $(a, \mathcal{F})$-singular asymptotic spectrum of u $v$ satisfies

$$
\begin{aligned}
\mathcal{S}_{\mathcal{A}}^{(\mathbf{a}, \mathcal{F})}(u v) \subset & \left\{(x, r) \in D_{1} \times \mathbb{R}_{+}^{n}, r \in \Sigma_{x}(u)\right\} \\
& \cup\left\{(x, r) \in D_{2} \times \mathbb{R}_{+}^{n}, r \in \Sigma_{x}(v)\right\} \\
& \cup\left\{(x, r) \in D_{3} \times \mathbb{R}_{+}^{n}, r \in E_{x}(u, v)\right\},
\end{aligned}
$$

where for any $x \in D_{3}$,

$$
E_{x}(u, v)=\mathbb{R}_{+}^{n} \backslash\left(N_{x}(u)+N_{x}(v)\right) .
$$

Remark 3 When e.g. $\Sigma_{x}(u)=\mathbb{R}_{+}^{n}$, then $N_{x}(u)=\emptyset$, and the sum $N_{x}(u)+N_{x}(v)$ also is empty, such that its complement is the whole of $\mathbb{R}_{+}^{n}$.

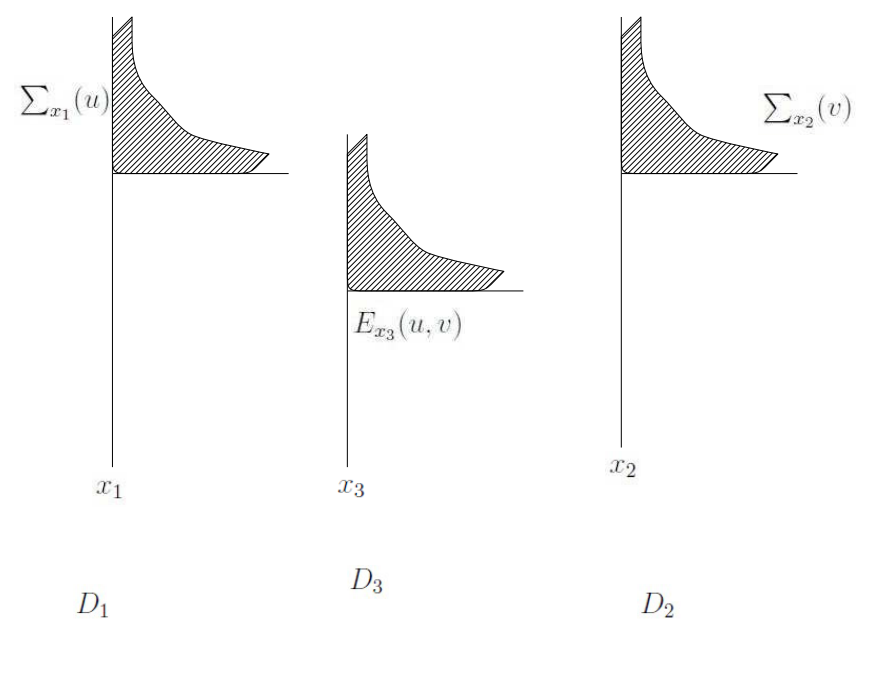

Proof. Since $\mathcal{F}$ is an algebra, the product $u v$ is $\mathcal{F}$-regular in all points in which $u$ and $v$ both are $\mathcal{F}$-regular, i.e. in all $x \notin \mathcal{S}_{\mathcal{A}}^{\mathcal{F}}(u) \cup \mathcal{S}_{\mathcal{A}}^{\mathcal{F}}(v)$. The points $x$ such that some $(x, r)$ might be in the $(\mathbf{a}, \mathcal{F})$-singular spectrum of $u v$ are thus contained in $D_{1} \cup D_{2} \cup D_{3}$.

Let us first consider $x \in D_{1}$, and $r \in N_{x}(u)$, i.e. $\left.\mathbf{a}(r) u\right|_{V} \in \mathcal{F}_{\mathcal{A}}(V)$ for some $V \in \mathcal{V}_{x}$. Then, since $v$ is $\mathcal{F}$-regular in $x$, there is $U \in \mathcal{V}_{x}$ such that $\left.v\right|_{U} \in \mathcal{F}_{\mathcal{A}}(U)$. Again using that $\mathcal{F}$ is a sheaf of algebras, we have $\mathbf{a}(r) u v \in \mathcal{F}_{\mathcal{A}}(U \cap V)$, and thus $r \in N_{x}(u v)$. This means that $\Sigma_{x}(u v) \subset \Sigma_{x}(u)$ for all $x \in D_{1}$. In the same way we get $\Sigma_{x}(u v) \subset \Sigma_{x}(v)$ for all $x \in D_{2}$.

Finally, let us consider $x \in D_{3}$, and $r \in N_{x}(u), s \in N_{x}(v)$, i.e., $\left.\mathbf{a}(r) u\right|_{U} \in \mathcal{F}_{\mathcal{A}}(U)$ and $\left.\mathbf{a}(s) v\right|_{V} \in \mathcal{F}_{\mathcal{A}}(V)$ for some $U, V \in \mathcal{V}_{x}$. Once again, since $\mathcal{F}_{\mathcal{A}}(U \cap V)$ is stable for the product, we have, $\left.\left.\mathbf{a}(r) u\right|_{W} \mathbf{a}(s) v\right|_{W}=\left.\mathbf{a}(r) \mathbf{a}(s)(u v)\right|_{W} \in \mathcal{F}_{\mathcal{A}}(W)$ for $W=U \cap V \in$ $\mathcal{V}_{x}$. By Definition 5, we have $\mathbf{a}(r+s)=O(\mathbf{a}(r) \mathbf{a}(s))$, and thus $\left.\mathbf{a}(r) \mathbf{a}(s)(u v)\right|_{W} \in$ $\left.\mathcal{F}_{\mathcal{A}}(W) \Rightarrow \mathbf{a}(r+s)(u v)\right|_{W} \in \mathcal{F}_{\mathcal{A}}(W)$. Otherwise said, we have $r+s \in N_{x}(u v)$, and thus, going to complements, $\Sigma_{x}(u, v) \subset \mathbb{R}_{+}^{n} \backslash\left(N_{x}(u)+N_{x}(v)\right)$ for all $x \in D_{3}$. 


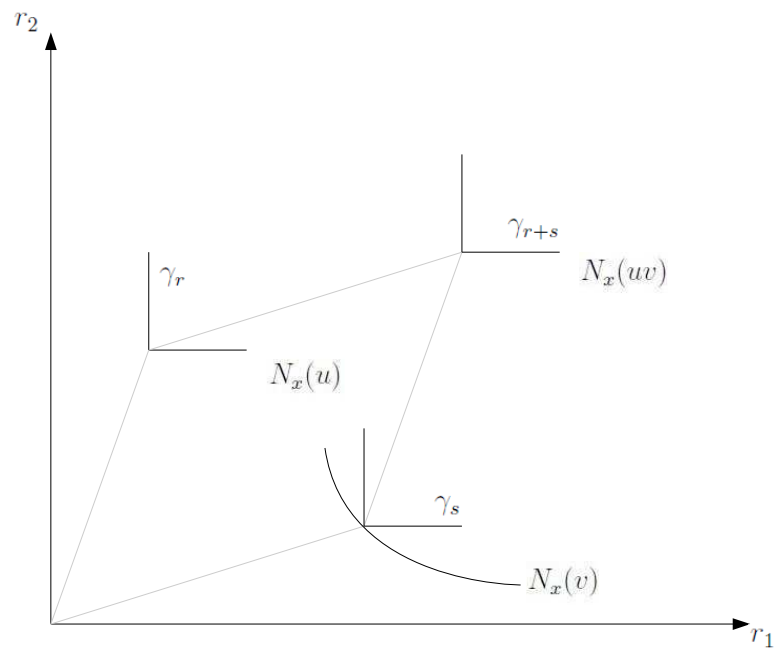

Corollary 7 For given $u \in \mathcal{A}(\Omega)$ and $p \in \mathbb{N}^{*}$, we have

$$
\mathcal{S}_{\mathcal{A}}^{(\mathbf{a}, \mathcal{F})}\left(u^{p}\right) \subset\left\{(x, r), x \in \mathcal{S}_{\mathcal{A}}^{\mathcal{F}}(u), r \in H_{p, x}(u)\right\}
$$

where

$$
H_{p, x}(u)=\mathbb{R}_{+}^{n} \backslash p N_{x}(u) .
$$

Remark 4 In the above, $p N_{x}(u)$ means $\left\{r_{1}+\cdots+r_{p} ; r_{1}, \ldots, r_{p} \in N_{x}(u)\right\}$, which is a larger set than $\left\{p r ; r \in N_{x}(u)\right\}$. The latter is contained in the former, so it would correspond to a weaker statement, which is implied by the above.

Proof. Using the preceding theorem, this follows by induction on $p$, where $D_{2}$ will be empty for $v=u^{p-1}$, since $\mathcal{S}_{\mathcal{A}}^{\mathcal{F}}\left(u^{p-1}\right) \subset \mathcal{S}_{\mathcal{A}}^{\mathcal{F}}(u)$.

Remark 5 In the above Corollary, $u^{p}$ could be replaced by any polynomial of degree $\leq p$ in $u$, with coefficients in $\mathcal{E}(\Omega)$. This follows indeed from the linear property eqn. (??) (which entails that we can deal with each monomial seperately) and from the fact that $H_{p}$ increases with $p$ (because $q \leq p \Rightarrow q N \supset p N$, for conic sets).

\subsection{Conic asymptotic regular fiber}

We can add some hypotheses rendering the asymptotic singular fiber the complement of a conic subset of $\mathbb{R}_{+}^{n}$.

Theorem 8 Consider

- $\Lambda=] 0,1]^{n} \ni \lambda=\left(\lambda_{1}, \lambda_{2}, . . \lambda_{n}\right)$

- $A$ overgenerated by the set $B_{n}=\left\{\left(\lambda_{1}\right)_{\lambda},\left(\lambda_{2}\right)_{\lambda}, \ldots,\left(\lambda_{n}\right)_{\lambda}\right\}$ 
- the map a from $\mathbb{R}_{+}^{n}$ to $A_{+}$defined by

$$
\mathbf{a}(r)=\mathbf{a}\left(r_{1}, r_{2}, \ldots, r_{n}\right)=\left(\lambda_{1}^{r_{1}} \lambda_{2}^{r_{2}} \cdots \lambda_{n}^{r_{n}}\right)_{\lambda}
$$

and let $u$ be a generalized function in $A(\Omega)$ where $\Omega$ is an open set in $X$, such that

$$
\exists p=\left(p_{1}, p_{2}, . . p_{n}\right) \in \mathbb{R}_{+}^{n}, \exists g \in \mathcal{F}(\Omega), g \neq 0, \lim _{\Lambda}{ }_{\mathcal{F}(V)} \mathbf{a}_{\lambda}(p) u_{\lambda}=g .
$$

Then, for each $x \in \operatorname{supp} g$, the $(\mathbf{a}, \mathcal{F})$-regular fiber is exactly the closed cone

$$
N_{x}(u)=\prod_{i=1}^{n}\left[p_{i},+\infty\right)
$$

with $p$ as apex. It follows that the the $(a, \mathcal{F})$-singular fiber is

$$
\Sigma_{x}(u)=\bigcup_{i=1,2, . . n}\left\{r \in \mathbb{R}_{+}^{n} \mid r_{i} \in\left[0, p_{i}\right)\right\} .
$$

Moreover, the above fibers are constant along $\operatorname{supp} g$.

Proof. By definition, $r=\left(r_{1}, r_{2}, . ., r_{n}\right)$ is in the fibre $N_{x}(u)$, if and only if,

$$
\exists V \in \mathcal{V}_{x} \quad \exists f \in \mathcal{F}(V): \lim _{\Lambda} \mathcal{F}(V)\left(\left.\mathbf{a}_{\lambda}(r) u_{\lambda}\right|_{V}\right)=f,
$$

i.e., if and only if,

$$
\exists V \in \mathcal{V}_{x} \quad \exists f \in \mathcal{F}(V): \lim _{\Lambda} \mathcal{F}(V)\left(\left.\mathbf{a}_{\lambda}(r-p) \Phi_{\lambda}\right|_{V}\right)=f,
$$

where $\Phi_{\lambda}=\mathbf{a}_{\lambda}(p) u_{\lambda}$. Since by assumption,

$$
\left.\lim _{\lambda} \mathcal{F}(V) \Phi_{\lambda}\right|_{V}=\left.g\right|_{V} \in \mathcal{F}(V) \text {, et }\left.g\right|_{V} \neq 0,
$$

the previous condition amounts to

$$
\lim _{\lambda} \mathbf{a}_{\lambda}(r-p)<+\infty .
$$

Let $s=r-p$. We want to show that the limit of $\mathbf{a}_{\lambda}(r-p)=\mathbf{a}_{\lambda}(s)=\lambda_{1}^{s_{1}} \lambda_{2}^{s_{2}} \cdots \lambda_{n}^{s_{n}}$, when $\left(\lambda_{1}, \lambda_{2}, . . \lambda_{n}\right)$ goes to $(0,0, \ldots, 0)$, can only be finite if $s_{i} \geq 0$ for all $i \in\{1,2, \ldots, n\}$. Indeed, assume that $s_{j}=\min \left\{s_{1}, \ldots, s_{n}\right\}<0$. Then it is sufficient to go to zero with

$$
\lambda_{i}=\varepsilon(i \neq j) ; \lambda_{j}=\varepsilon^{k}
$$

where $k$ is chosen large enough such that $-k s_{j}>\left|s_{1}\right|+\left|s_{2}\right|+\ldots+\left|s_{n}\right|=:|s|_{1}$.

Then clearly,

$$
\lambda_{1}^{s_{1}} \lambda_{2}^{s_{2}} \cdots \lambda_{n}^{s_{n}} \geq \varepsilon^{|s|_{1}+k s_{j}} \rightarrow+\infty \quad(\varepsilon \rightarrow 0) .
$$

Thus, we must have $s_{i} \geq 0$ for all $i \in\{1, \ldots, n\}$, i.e., $r_{1} \geq p_{1}, r_{2} \geq p_{2}, \ldots, r_{n} \geq p_{n}$. This means, the regular fibre is exactly the cone

$$
N_{x}(u)=\prod_{i=1}^{n}\left[p_{i},+\infty\right)
$$




\section{A transport equation with irregular coefficients and data}

Here we are interested in the study of the Cauchy problem for a linear transport equation where the coefficients $\alpha$ and $\beta$ and the data $u_{0}$ are irregular and even distributions (or more singular objects).

$$
(P)\left\{\begin{array}{r}
\frac{\partial}{\partial t} u(t, x, y)+\alpha(t) \frac{\partial}{\partial x} u(t, x, y)+\beta(t) \frac{\partial}{\partial y} u(t, x, y)=0 \\
u(0, x, y)=u_{0}(x, y) .
\end{array}\right.
$$

We recall that if $\alpha, \beta \in \mathrm{C}^{\infty}(\mathbb{R})$ and $u_{0} \in \mathrm{C}^{\infty}\left(\mathbb{R}^{2}\right)$, when putting $f(t)=\int_{0}^{t} \alpha(\tau) \mathrm{d} \tau$, $g(t)=\int_{0}^{t} \beta(\tau) \mathrm{d} \tau$, we have the smooth solution

$$
u(t, x, y)=u_{0}(x-f(t), y-g(t))
$$

which propagates the data along the caracteristic curve $\Gamma=\{t=s, x=f(s), y=g(s)\}$.

If $u_{0}=v \in \mathcal{D}^{\prime}\left(\mathbb{R}^{2}\right)$, and always $\alpha, \beta \in \mathrm{C}^{\infty}(\mathbb{R})$, we still can formulate the problem as

$$
\left(P_{\text {dist }}\right)\left\{\begin{array}{c}
\frac{\partial}{\partial t} u+\left(\alpha \otimes 1_{x y}\right) \frac{\partial}{\partial x} u+\left(\beta \otimes 1_{x y}\right) \frac{\partial}{\partial y} u(t, x, y)=0, \\
\left.u\right|_{\{t=0\}}=v .
\end{array}\right.
$$

The problem is well posed in $\mathcal{D}^{\prime}\left(\mathbb{R}^{3}\right)$ (see [3]) and admits as solution the distribution $v_{\Gamma}$ defined for $\psi \in \mathcal{D}\left(\mathbb{R}^{3}\right)$ by

$$
\left\langle v_{\Gamma}, \psi\right\rangle=\left\langle v,(x, y) \mapsto \int \psi(t, x+f(t), y+g(t)) \mathrm{d} t\right\rangle .
$$

In particular, if $v=\delta$, the solution is the Dirac measure on the characteristic curve

$$
\left\langle\delta_{\Gamma}, \psi\right\rangle=\int \psi(t, f(t), g(t)) \mathrm{d} t .
$$

Now in the case where the coefficients are distributions and the data a more singular object $\delta_{x}^{p} \otimes \delta_{y}^{q}$ to which we will give later a generalized meaning, the problem is formally written as

$$
\left(P_{\text {form }}\right)\left\{\begin{array}{c}
\frac{\partial}{\partial t} u+\left(\alpha \otimes 1_{x y}\right) \frac{\partial}{\partial x} u+\left(\beta \otimes 1_{x y}\right) \frac{\partial}{\partial y} u=0, \\
\left.u\right|_{\{t=0\}}=\delta_{x}^{p} \otimes \delta_{y}^{q} .
\end{array}\right.
$$

The coefficients $A=\alpha \otimes 1_{x y}$ and $B=\beta \otimes 1_{x y}$ are now distributions in $\mathcal{D}^{\prime}\left(\mathbb{R}^{3}\right)$ but generally the factor $A \frac{\partial}{\partial x} u+B \frac{\partial}{\partial y} u$ has no meaning in $\mathcal{D}^{\prime}\left(\mathbb{R}^{3}\right)$, and it is the same for $\delta_{x}^{p} \otimes \delta_{y}^{q}$ except for $p=q=1$.

\subsection{Solving the associated generalized problem}

We can associate to $\left(P_{\text {form }}\right)$ a generalized problem, well formulated in a convenient $(\mathcal{C}, \mathcal{E}, \mathcal{P})$-algebra $\mathcal{A}\left(\mathbb{R}^{3}\right)$, as follows. 
Take $\varphi, \psi, \theta \in \mathcal{D}(\mathbb{R})$ such that $\int \varphi(x) d x=\int \psi(y) \mathrm{d} y=\int \theta(t) \mathrm{d} t=1$. Then let $\varphi_{\varepsilon}=\frac{1}{\varepsilon} \varphi\left(\frac{\dot{\varepsilon}}{\varepsilon}\right), \psi_{\eta}=\frac{1}{\eta} \psi\left(\frac{\dot{\eta}}{\eta}\right), \theta_{\rho}=\frac{1}{\rho} \psi(\dot{\dot{\rho}})$. Now set $\alpha_{\rho}=\alpha * \theta_{\rho}, \beta_{\rho}=\beta * \theta_{\rho}$. We consider $\mathcal{C}=A / I$ and the associated spaces $\mathcal{A}\left(\mathbb{R}^{3}\right)$ and $\mathcal{A}\left(\mathbb{R}^{2}\right)$ overgenerated by

$$
\left\{(\varepsilon)_{\varepsilon, \eta, \rho},(\eta)_{\varepsilon, \eta, \rho},(\rho)_{\varepsilon, \eta, \rho}\right\}
$$

Finally, we define $H_{\varepsilon, \eta}(x, y)=\varphi_{\varepsilon}^{p}(x) \psi_{\eta}^{q}(y), F_{\rho}=\alpha_{\rho} \otimes 1_{x y}, G_{\rho}=\beta_{\rho} \otimes 1_{x y}$.

It is clear that $\left(F_{\rho}\right)_{\varepsilon, \eta, \rho}$ (resp. $\left.\left(G_{\rho}\right)_{\varepsilon, \eta, \rho}\right)$ is a representative of some $F$ (resp. $G$ ) belonging to $\mathcal{A}\left(\mathbb{R}^{3}\right)$ and $\left(H_{\varepsilon, \eta}\right)_{\varepsilon, \eta, \rho}$ is a representative of some $H$ belonging to $\mathcal{A}\left(\mathbb{R}^{2}\right)$. Thus $F$ and $G$ (resp. $H$ ) are the classes in $\mathcal{A}\left(\mathbb{R}^{3}\right)$ (resp. $\left.\mathcal{A}\left(\mathbb{R}^{2}\right)\right)$ of the families regularizing the coefficients (resp. the data). Then

$$
\left(P_{\text {gen }}\right)\left\{\begin{array}{c}
\frac{\partial}{\partial t} u+F \frac{\partial}{\partial x} u+G \frac{\partial}{\partial y} u=0 \\
\left.u\right|_{\{t=0\}}=H .
\end{array}\right.
$$

is well formulated.

To solve $\left(P_{g e n}\right)$ we first solve

$$
\left(P_{\infty}\right)\left\{\begin{array}{c}
\frac{\partial}{\partial t} u_{\varepsilon, \eta, \rho}+a_{\rho}(t) \frac{\partial}{\partial x} u_{\varepsilon, \eta, \rho}+b_{\rho}(t) \frac{\partial}{\partial y} u_{\varepsilon, \eta, \rho}=0 \\
u_{\varepsilon, \eta, \rho}(0, x, y)=H_{\varepsilon, \eta}(x, y)
\end{array}\right.
$$

with solution

$$
u_{\varepsilon, \eta, \rho}(t, x, y)=H_{\varepsilon, \eta}\left(x-\Phi_{\rho}(t), y-\Psi_{\rho}(t)\right)
$$

where $\Phi_{\rho}(t)=\int_{0}^{t} a_{\rho}(\tau) \mathrm{d} \tau, \Psi_{\rho}(t)=\int_{0}^{t} b_{\rho}(\tau) \mathrm{d} \tau$.

We shall use the following lemmas.

Lemma 9 Set $f, g \in \mathrm{C}^{\infty}\left(\mathbb{R}^{3}, \mathbb{R}\right), F \in \mathrm{C}^{\infty}\left(\mathbb{R}^{2}, \mathbb{R}\right), \mathcal{U}(t, x, y)=H(f(t, x, y), g(t, x, y))$. For any $n \geq 0, m \geq 0, p \geq 0, \alpha=(n, m, p)$, with $|\alpha|=n+m+p>1$, we have

$$
\begin{aligned}
\frac{\partial^{n+m+p} \mathcal{U}}{\partial t^{n} \partial x^{m} \partial^{p} y}(t, x, y)= & \sum_{1 \leq|\beta| \leq n+m+p} c_{\beta}\left(D^{\beta} H\right)(f(t, x, y), g(t, x, y)) \times \\
& \times \sum_{i=1}^{n+m+p} \sum_{p_{i}(\alpha, \beta)} d_{i, \alpha, \beta} \prod_{j=1}^{i}\left(D^{l_{j}} f(t, x, y)\right)^{k_{j, 1}}\left(D^{l_{j}} g(t, x, y)\right)^{k_{j, 2}}
\end{aligned}
$$

where $\beta \in \mathbb{N}^{2}, c_{\beta} \geq 0, d_{i, \alpha, \beta} \geq 0$. The set $p_{i}(\alpha, \beta)$ mentioned in the inner sum consists of all nonzero multi-indices $\left(k_{1}, \ldots, k_{i}, l_{1}, \ldots, l_{i}\right) \in\left[(\mathbb{N})^{2}\right]^{i} \times\left[(\mathbb{N})^{3}\right]^{i}$, such that

$$
0 \prec l_{1} \prec \ldots \prec l_{i}, \quad \sum_{j=1}^{i} k_{j}=\beta, \quad \sum_{j=1}^{i}\left|k_{j}\right| l_{j}=\alpha,
$$


where the linear order on $\mathbb{N}^{3}$ is defined as follows: if $\mu=\left(\mu_{1}, \mu_{2}, \mu_{3}\right)$ and $\nu=\left(\nu_{1}, \nu_{2}, \nu_{3}\right)$ are in $\mathbb{N}^{3}, \mu \prec \nu$ provided one of the following holds

$$
\left\{\begin{array}{c}
|\mu|<|\nu| ; \\
|\mu|=|\nu| \text { and } \mu_{1}<\nu_{1} \\
|\mu|=|\nu|, \mu_{1}=\nu_{1} \text { and } \mu_{2}<\nu_{2} \\
|\mu|=|\nu|, \mu_{1}=\nu_{1}, \mu_{2}=\nu_{2} \text { and } \mu_{3}<\nu_{3}
\end{array}\right.
$$

The proof uses the Multivariate Faà di Bruno's formula (see [2]).

Theorem 10 The class of $\left(u_{\varepsilon, \eta, \rho}\right)_{\varepsilon, \eta, \rho}$ in $\mathcal{A}\left(\mathbb{R}^{3}\right)$ is solution of $\left(P_{\text {gen }}\right)$.

Proof. Taking $u_{\varepsilon, \eta, \rho}(t, x, y)=H_{\varepsilon, \eta}\left(x-\Phi_{\rho}(t), y-\Psi_{\rho}(t)\right), u_{\varepsilon, \eta, \rho}$ is solution to $\left(P_{\infty}\right)$. We must prove that the family $\left(u_{\varepsilon, \eta, \rho}\right)_{\varepsilon, \eta, \rho}$ lies in $\mathcal{A}\left(\mathbb{R}^{3}\right)$ through technical estimates proving that $\left(p_{L, l}\left(u_{\varepsilon, \eta, \rho}\right)\right)_{\varepsilon, \eta, \rho} \in|A|$, for the family of usual semi-norms $p_{L, l}$ describing the topology of $\left.\mathrm{C}^{\infty}\left(\mathbb{R}^{3}\right)\right)$. Then $u=\left[u_{\varepsilon, \eta, \rho}\right]$ is a solution to $\left(P_{g e n}\right)$.

To simplify, suppose that

$$
\exists K \Subset \mathbb{R}^{2}, \forall \varepsilon, \forall \eta, \operatorname{supp} H_{\varepsilon, \eta} \subset K,
$$

(for example, suppose that $\operatorname{supp} \varphi$ and $\operatorname{supp} \psi$ are compact). Take $L \Subset \mathbb{R}^{3}$,

$$
\begin{aligned}
\left(p_{L, 0}\left(u_{\varepsilon, \eta, \rho}\right)\right)_{\varepsilon, \eta, \rho} & =\left(\sup _{(t, x, y) \in L}\left|H_{\varepsilon, \eta}\left(x-\Phi_{\rho}(t), y-\Psi_{\rho}(t)\right)\right|\right)_{\varepsilon, \eta, \rho} \\
& \leq\left(\sup _{(\xi, \varsigma) \in K}\left|H_{\varepsilon, \eta}(\xi, \varsigma)\right|\right)_{\varepsilon, \eta}=\left(P_{K, 0}\left(H_{\varepsilon, \eta}\right)\right)_{\varepsilon, \eta} \in|A| .
\end{aligned}
$$

As the derivatives with respect to $x$ (resp. with respect to $y$ ) have the same support, we obtain similar estimates,

$$
\left(\sup _{(t, x, y) \in L}\left|\frac{\partial^{l}}{\partial x^{l}} H_{\varepsilon, \eta}\left(x-\Phi_{\rho}(t), y-\Psi_{\rho}(t)\right)\right|\right)_{\varepsilon, \eta, \rho} \leq\left(P_{K, l}\left(H_{\varepsilon, \eta}\right)\right)_{\varepsilon, \eta} \in|A| .
$$

Using Lemma 9, with $f(t, x, y)=\left(x-\Phi_{\rho}(t), g(t, x, y)=\left(y-\Psi_{\rho}(t)\right)\right.$, we have

$$
\begin{aligned}
\frac{\partial^{n+m+p} u_{\varepsilon, \eta, \rho}}{\partial t^{n} \partial x^{m} \partial^{p} y}(t, x, y)= & \sum_{1 \leq|\beta| \leq n+m+p} c_{\beta}\left(D^{\beta} H_{\varepsilon, \eta}\right)(f(t, x, y), g(t, x, y)) \times \\
& \times \sum_{i=1}^{n+m+p} \sum_{p_{i}(\alpha, \beta)} d_{i, \alpha, \beta} \prod_{j=1}^{i}\left(D^{l_{j}} f(t, x, y)\right)^{k_{j, 1}}\left(D^{l_{j}} g(t, x, y)\right)^{k_{j, 2}} .
\end{aligned}
$$

Moreover

$$
D^{l_{j}} f(t, x, y)=\left\{\begin{array}{cl}
-\Phi_{\rho}^{\left(l_{j, 1}\right)}(t) & \text { if } l_{j}=\left(l_{j, 1}, 0,0\right) \\
1 & \text { if } l_{j}=(0,1,0) \\
0 & \text { else. }
\end{array}\right.
$$


and

$$
D^{l_{j}} g(t, x, y)=\left\{\begin{array}{cl}
-\Psi_{\rho}^{\left(l_{j, 1}\right)}(t) & \text { if } l_{j}=\left(l_{j, 1}, 0,0\right) \\
1 & \text { if } l_{j}=(0,1,0), \\
0 & \text { else. }
\end{array}\right.
$$

Then we have

$$
\begin{aligned}
& \left|D^{\alpha} u_{\varepsilon, \eta, \rho}(t, x, y)\right| \\
& \leq \sum_{1 \leq|\beta| \leq|\alpha|} c_{\beta} P_{K,|\alpha|}\left(H_{\varepsilon, \eta}\right) \sum_{i=1}^{|\alpha|} \sum_{p_{i}(\alpha, \beta)} d_{i, \alpha, \beta} \prod_{j=1}^{i}\left(P_{K, l_{j, 1}}\left(\Phi_{\rho}\right)\right)^{k_{j, 1}}\left(P_{K, l_{j, 1}}\left(\Psi_{\rho}\right)\right)^{k_{j, 21}}
\end{aligned}
$$

Hence we can find a constant $C_{\alpha}$, independent of $H_{\varepsilon, \eta}$ and $u_{\varepsilon, \eta, \rho}$, such that,

$$
P_{L,|\alpha|}\left(u_{\varepsilon, \eta, \rho}\right) \leq C_{\alpha} P_{K,|\alpha|}\left(H_{\varepsilon, \eta}\right)\left(P_{K, n}\left(\Phi_{\rho}\right)\right)^{q}\left(P_{K, n}\left(\Psi_{\rho}\right)\right)^{r},
$$

with $q+r \leq|\alpha|$. This will be estimated by an expression of the form $C \varepsilon^{-N} \eta^{-M} \rho^{-P}$, for $\varepsilon, \eta, \rho$ small enough. Since $A$ is a solid ring, we will have

$$
\left(P_{L,|\alpha|}\left(u_{\varepsilon, \eta, \rho}\right)\right)_{\varepsilon, \eta, \rho} \in|A| \text {. }
$$

The result of this is that

$$
\forall L \Subset \mathbb{R}^{3}, \forall l \in \mathbb{N},\left(P_{L, l}\left(u_{\varepsilon, \eta, \rho}\right)\right)_{\varepsilon, \eta, \rho} \in|A| .
$$

Then $\left(u_{\varepsilon, \eta, \rho}\right)_{\varepsilon, \eta, \rho} \in \mathcal{A}\left(\mathbb{R}^{3}\right)$ and $u=\left[u_{\varepsilon, \eta, \rho}\right]$ is a solution to $\left(P_{g e n}\right)$.

\subsection{Local and microlocal asymptotic analysis of the solution}

In our case, we are going to prove that the $\mathcal{D}^{\prime}$-singularities of the data propagate along the "regularized characteristic $\Gamma$ of the problem $\left(\mathcal{P}_{g e n}\right)$ " on which the fiber $\Sigma_{X}(u)$ remains constant. We begin to prove the main following lemma which provides an example of hypothesis used in Theorem 8 when $\mathcal{F}=\mathcal{D}^{\prime}$.

Lemma 11 Suppose that $\lim _{\rho \rightarrow 0} \Phi_{\rho}=\Phi, \lim _{\rho \rightarrow 0} \Psi_{\rho}=\Psi$ in the $L_{\text {loc }}^{1}(\mathbb{R})$ topology and let us consider the family

$$
v_{\varepsilon, \eta, \rho}(t, x, y)=\frac{1}{\varepsilon} \frac{1}{c_{p}} \varphi^{p}\left(\frac{x-\Phi_{\rho}(t)}{\varepsilon}\right) \frac{1}{\eta} \frac{1}{c_{q}^{\prime}} \psi^{q}\left(\frac{y-\Psi_{\rho}(t)}{\eta}\right)
$$

where $c_{p}=\int \varphi^{p}(x) \mathrm{d} x, c_{q}^{\prime}=\int \psi^{q}(x) \mathrm{d} x$. Then

$$
\lim _{\substack{(\varepsilon, \eta, \rho) \rightarrow(0,0,0) \\ \mathcal{D}^{\prime}\left(\mathbb{R}^{3}\right)}} v_{\varepsilon, \eta, \rho}=\delta_{\Gamma}
$$

where $\delta_{\Gamma}$ is the Dirac distribution of the manifold $\Gamma=\{(t, x, y): x=\Phi(t), y=\Psi(t)\}$. 
Proof. Let $\delta_{\Gamma}\left(\operatorname{resp} . \delta_{\Gamma_{\rho}}\right)$ be the Dirac distribution of the manifold $\Gamma\left(\operatorname{resp} . \Gamma{ }_{\rho}\right)$, defined by,

$$
\left\langle\delta_{\Gamma}, \theta\right\rangle=\int \theta(t, \Phi(t), \Psi(t)) \mathrm{d} t,\left(\operatorname{resp} .\left\langle\delta_{\Gamma_{\rho}}, \theta\right\rangle=\int \theta\left(t, \Phi_{\rho}(t), \Psi_{\rho}(t)\right) \mathrm{d} t\right),
$$

for any $\theta \in \mathcal{D}\left(\mathbb{R}^{3}\right)$.

If $(\varepsilon, \eta, \rho)$ tends to $(0,0,0)$ in any way (for the usual topology), then

$$
\lim _{\substack{(\varepsilon, \eta, \rho) \rightarrow(0,0,0) \\ \mathcal{D}^{\prime}\left(\mathbb{R}^{3}\right)}} v_{\varepsilon, \eta, \rho}=\lim _{\rho \rightarrow 0}\left(\lim _{\substack{(\varepsilon, \eta) \rightarrow(0,0) \\ \mathcal{D}^{\prime}\left(\mathbb{R}^{3}\right)}} v_{\varepsilon, \eta, \rho}\right) .
$$

Classical computations show that: $\lim _{\substack{(\varepsilon, \eta) \rightarrow(0,0) \\ \mathcal{D}^{\prime}\left(\mathbb{R}^{3}\right)}} v_{\varepsilon, \eta, \rho}=\delta_{\Gamma_{\rho}}$ and then we are going to show that $\lim _{\rho \rightarrow 0}\left(\lim _{\substack{(\varepsilon, \eta) \rightarrow(0,0) \\ \mathcal{D}^{\prime}\left(\mathbb{R}^{3}\right)}} v_{\varepsilon, \eta, \rho}\right)=\delta_{\Gamma}$ by computing $\lim _{\rho \rightarrow 0}\left(\delta_{\Gamma_{\rho}}-\delta_{\Gamma}\right)$. We have

$$
\left\langle\left(\delta_{\Gamma_{\rho}}-\delta_{\Gamma}\right), \theta\right\rangle=\int_{K}\left(\theta\left(t, \Phi_{\rho}(t), \Psi_{\rho}(t)\right)-\theta(t, \Phi(t), \Psi(t))\right) \mathrm{d} t
$$

with $K=\operatorname{proj}_{t}(\operatorname{supp} \theta)$, then

$$
\left|\left\langle\left(\delta_{\Gamma_{\rho}}-\delta_{\Gamma}\right), \theta\right\rangle\right|=\int_{K}\left(\left|\Phi_{\rho}(t)-\Phi(t)\right|\left|\frac{\partial \theta}{\partial x}\left(h_{\rho}(t)\right)\right|+\left|\Psi_{\rho}(t)-\Psi(t)\right|\left|\frac{\partial \theta}{\partial y}\left(k_{\rho}(t)\right)\right|\right) \mathrm{d} t,
$$

for some $h_{\rho}(t)$ and $k_{\rho}(t)$. But $\frac{\partial \theta}{\partial x}$ and $\frac{\partial \theta}{\partial y}$ being respectively bounded by $M$ and $N$, we have

$$
\left|\left\langle\left(\delta_{\Gamma_{\rho}}-\delta_{\Gamma}\right), \theta\right\rangle\right| \leq M \int_{K}\left|\Phi_{\rho}(t)-\Phi(t)\right| d t+N \int_{K}\left|\Psi_{\rho}(t)-\Psi(t)\right| \mathrm{d} t
$$

which gives the result from the hypothesis.

Theorem 12 (Asymptotic singular support)

(i) If $p=1$ and $q=1$, the $\mathcal{D}^{\prime}$-singular support of $u=\left[u_{\varepsilon, \eta, \rho}\right]$ is the empty set.

(ii) If $p>1$ or $q>1$, the $\mathcal{D}^{\prime}$-singular support of $u=\left[u_{\varepsilon, \eta, \rho}\right]$ is the closure $\bar{\Gamma}$ of the "regularized characteristic" curve

$$
\Gamma=\{(t, x, y): x=\Phi(t), y=\Psi(t)\} .
$$

Proof. We have

$$
\begin{aligned}
u_{\varepsilon, \eta, \rho}(t, x, y) & =H_{\varepsilon, \eta}\left(x-\Phi_{\rho}(t), y-\Psi_{\rho}(t)\right)=\varphi_{\varepsilon}^{p}\left(x-\Phi_{\rho}(t)\right) \psi_{\eta}^{q}\left(y-\Psi_{\rho}(t)\right) \\
& =\frac{c_{p}}{\varepsilon^{p-1}} \frac{c_{q}^{\prime}}{\eta^{q-1}} v_{\varepsilon, \eta, \rho}(t, x, y)
\end{aligned}
$$

where $v_{\varepsilon, \eta, \rho}(t, x, y)$ is just defined in the previous lemma. 
If $p=q=1$, for each $X=(t, x, y) \in \mathbb{R}^{3} \backslash \bar{\Gamma}$ (resp. $\bar{\Gamma}$ ) it exists a neighbourhood $V$ of $X$ such that $\left.u_{\varepsilon, \eta, \rho}\right|_{V}=0$ for all $\varepsilon, \eta, \rho$ small enough (resp. $\left.\lim _{\substack{\varepsilon, \eta, \rho) \rightarrow(0,0,0) \\ \mathcal{D}^{\prime}\left(\mathbb{R}^{3}\right)}} u_{\varepsilon, \eta, \rho}\right|_{V} \in$ $\left.\mathcal{D}^{\prime}(V)\right)$. Thus the $\mathcal{D}^{\prime}$-singular support of $u$ is the empty set.

If $p>1$ or $q>1, u$ is always equal to 0 in some neighbourhood of each $X \in \mathbb{R}^{3} \backslash \bar{\Gamma}$. But in any neighbourhood $V$ of each $X \in \bar{\Gamma},\left.u_{\varepsilon, \eta, \rho}\right|_{V}$ has no limit in $\mathcal{D}^{\prime}(V)$, thus the $\mathcal{D}^{\prime}$-singular support of $u$ is $\bar{\Gamma}$.

Theorem 13 (Asymptotic singular spectrum)

For $r=\left(r_{1}, r_{2}, r_{3}\right)$, choose $\mathbf{a}(r)=\left[\left(\varepsilon^{r_{1}} \eta^{r_{2}} \rho^{r_{3}}\right)_{(\varepsilon, \eta, \rho)}\right]$.

(i) If $p=1$ and $q=1$, then the $\left(\mathbf{a}, \mathcal{D}^{\prime}\right)$-singular spectrum of $u \in \mathcal{A}\left(\mathbb{R}^{3}\right)$ is the empty set.

(ii) If $p>1$ and $q>1$, then the $\left(\mathbf{a}, \mathcal{D}^{\prime}\right)$-singular spectrum of $u \in \mathcal{A}\left(\mathbb{R}^{3}\right)$ is the set

$$
\begin{aligned}
\mathcal{S}_{\mathcal{A}}^{\left(a, \mathcal{D}^{\prime}\right)}(u) & =\left\{(X, r) \in \mathbb{R}^{3} \times \mathbb{R}_{+}^{3} \mid X=(t, x, y) \in \bar{\Gamma}, r \in \Sigma_{X}(u)\right\}, \\
\Sigma_{X}(u) & =\left\{r \in \mathbb{R}_{+}^{3} \mid r_{1} \in\left[0, p-1\left[\vee r_{2} \in[0, q-1[\} .\right.\right.\right.
\end{aligned}
$$

The singularities of the data propagate along the "regularized characteristic" $\Gamma$ on which the fiber $\Sigma_{X}(u)$ is constant.

Proof. If $p=1$ and $q=1$, the $\mathcal{D}^{\prime}$-singular support of $u=\left[u_{\varepsilon, \eta, \rho}\right]$ is the empty set from the previous theorem, and it is obviously the same for the $\left(\mathbf{a}, \mathcal{D}^{\prime}\right)$-singular spectrum of $u$.

Suppose $p>1$ and $q>1$. We have

$$
\lim _{\substack{(\varepsilon, \eta, \rho) \rightarrow(0,0,0) \\ \mathcal{D}^{\prime}\left(\mathbb{R}^{3}\right)}} \mathbf{a}_{\varepsilon, \eta, \rho}(p-1, q-1,0) u_{\varepsilon, \eta, \rho}=\lim _{\substack{(\varepsilon, \eta, \rho) \rightarrow(0,0,0) \\ \mathcal{D}^{\prime}\left(\mathbb{R}^{3}\right)}} c_{p} c_{q}^{\prime} v_{\varepsilon, \eta, \rho}=c_{p} c_{q}^{\prime} \delta_{\Gamma} .
$$

According to Theorem 8, above each $X \in \operatorname{supp} \delta_{\Gamma}\left(=S_{\mathcal{A}}^{\mathcal{D}^{\prime}}(u)\right)$, the singular fiber is

$$
\Sigma_{X}(u)=\left\{r: r_{1} \in\left[0, p-1[\} \cup\left\{r: r_{2} \in[0, q-1[\} .\right.\right.\right.
$$

Thus the $\left(\mathbf{a}, \mathcal{D}^{\prime}\right)$-singular spectrum of $u \in \mathcal{A}\left(\mathbb{R}^{3}\right)$ is the set

$$
\mathcal{S}_{\mathcal{A}}^{\left(\mathbf{a}, \mathcal{D}^{\prime}\right)}(u)=\left\{(X, r) \in \mathbb{R}^{3} \times \mathbb{R}_{+}^{3} \mid X=(t, x, y) \in \bar{\Gamma}, r \in \Sigma_{X}(u)\right\} .
$$

Remark 6 The singular fiber $\Sigma_{X}(u)$ becomes smaller when the singularities of the data decrease. For example, in the previous result, when taking $p=1$ and $q=1$, the $\mathcal{D}^{\prime}$-singular fiber $\Sigma_{X}(u)$ is the empty set.

Remark 7 If we choose the distribution coefficients $\alpha$ and $\beta$ as the Heaviside function $Y$ (with the same data), the singular fiber $\Sigma_{X}(u)$ is described in Theorem 13, $X$ running over the line

$$
\Gamma=\{(t, x, y): x=y=t Y(t)\}
$$


which is also the $\mathcal{D}^{\prime}$-singular support of $u$.

If the coefficients are choosen as the Dirac $\delta$, the singular fiber is given by the same expression, but $X$ runs over the $\mathcal{D}^{\prime}$-singular support of $u$,

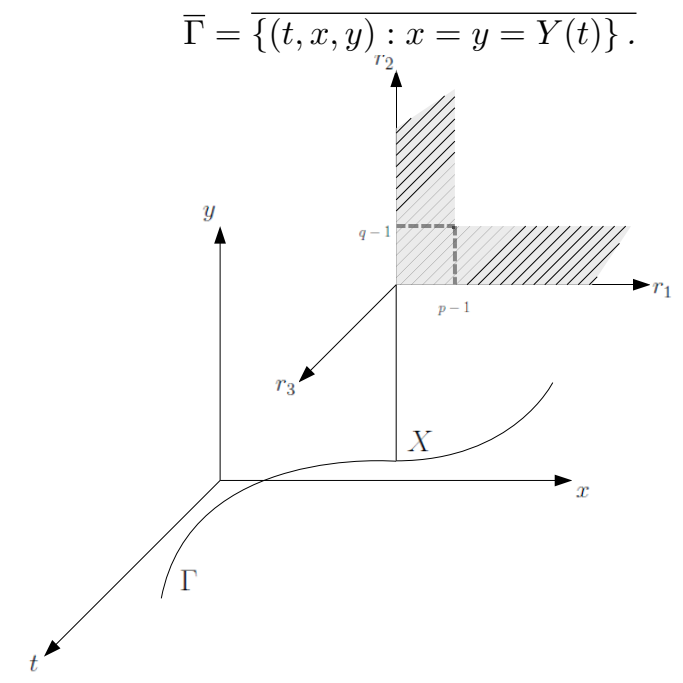

\section{References}

[1] N. Caroff, Generalized solutions of linear partial differential equations with discontinuous coefficients. Differential Integral Equations 17/5-6, 653-668, (2004).

[2] G. M. Constantine, H. Savits, A multivariate Faa di Bruno formula with applications. Trans. of the A. M. S. 348(2) (1996), 503-520.

[3] A. Delcroix, V. Dévoué, J.-A. Marti, Generalized solutions of singular differential problems. Relationship with classical solutions. J. Math. Anal. Appl. 353, 386-402, (2009).

[4] A. Delcroix, J.-A. Marti, M. Oberguggenberger, Spectral Asymptotic Analysis in Algebras of Generalized Functions. Asymptotic Analysis 59/1-2, 83-107, (2008). DOI:10.3233/ASY-2008-0885

[5] M. F. Hasler, Asymptotic extension of topological modules and algebras. Int. Trans. Spec. Funct., 20/3-4, 291-299, (2009).

[6] C. Le Bris, P.-L. Lions, Existence and Uniqueness of solutions to Fokker-Planck type Equations with Irregular Coefficients. Comm. in PDE, 33/7, 1272-1317 (2008).

[7] J.-A. Marti, $(C, E, P)$-Sheaf structure and applications, in: Nonlinear Theory of Generalized Functions, Chapman \& Hall/CRC Research Notes in Mathematics 401, M. Grosser, G. Hörmann, M. Kunzinger and M. Oberguggenberger, eds., Boca Raton (1999), pp. 175-186. 
[8] J.-A. Marti, Nonlinear algebraic analysis of delta shock wave solutions to Burgers' equation. Pacific Journal of Mathematics 210, 165-187 (2003).

[9] J.-A. Marti, Regularity, Local and Microlocal Analysis in Theories of Generalized Functions. Act. Appl. Math. 105, 267-302 (2009). 